\title{
APPLICATION OF X-RAY COMPUTED TOMOGRAPHY IN SILICON SOLAR CELLS
}

\author{
V.A. Popovich ${ }^{1}$, W. Verwaal ${ }^{2}$, M. Janssen ${ }^{1}$, I. J. Bennett ${ }^{3}$, I.M.Richardson ${ }^{1}$, \\ 1. Delft University of Technology, Department of Materials Science \& Engineering, \\ Delft, The Netherlands, Phone: +31 (0) 1527895 68, email: v.popovich@tudelft.nl \\ 2. Delft University of Technology, Department of Geotechnology, Delft, The Netherlands, \\ 3. Energy Research Centre of the Netherlands, Solar Energy, PV Module Technology, \\ Petten, The Netherlands
}

\begin{abstract}
The present study outlines the characterization of the internal microstructure in a multicrystalline silicon solar cell, by means of a powerful non-intrusive experimental method, namely X-ray computed tomography. The purpose of this research is to give a better understanding of the silicon solar cells metallization layers and defects related to its processing. Resulting tomographic images showed the distribution of bismuth glass and porosity in Al and $\mathrm{Ag}$ contact layers. At the same time, 3D tomographic images revealed the presence of process induced defects.

In this work the usefulness of the CT technique for the in depth study of silicon solar cells is shown.
\end{abstract}

\section{INTRODUCTION}

Nowadays solar cells and solar panels represent an interconnection system with different interfaces in a multi-layer/multistacked package, resulting from a complex multi-step production process. The most critical processing step during the manufacture of screen-printed solar cells is the firing of metallic contacts. Residual stresses are formed within the cell due to mismatch of thermal expansion coefficients and different mechanical behavior of the materials used in the metallic contacts. The wafer bows and forms a convex or concave body upon cooling, which mechanically loads the cell and may cause fracture. Nowadays it is very important to find a compromise between electrical properties, strength and costs of the solar cell. To achieve this, it is necessary to have a better understanding of the microstructure and mechanical properties of the cell.

Aluminum and silver screen-printed metallic contacts are non-homogeneous materials and their macroscopic physical and mechanical properties depend on their local characteristics (physical properties of elementary components, micro-geometry, porosity and defects), which at the end affect the mechanical behavior of the entire solar cell. Thus, it is of importance to link the local and the macroscopic scale in order to study and model the mechanical behavior of solar cells. This requires the knowledge of the 3D micro-geometry. The photovoltaic industry experiences a lack of non-destructive characterization tools, that could allow qualitative and quantitative analysis of internal features of solar cells and solar panels.

X-ray computed tomography (CT) is a nondestructive technique that allows visualization of the internal structure of objects, determined mainly by variations in density and atomic composition. It requires the acquisition of one or two-dimensional radiographs for different positions during step-wise rotation around a central axis, whereby either the source and detector or the sample are moved. The basic principle of CT is summarized in [1, 2].

In this work the usefulness of the CT technique for the in-depth study of silicon solar cells is shown. The purpose of this research is to give a better understanding of the silicon solar cells metallization layers and defects related to its processing.

\section{EXPERIMENTAL CONDITIONS}

\subsection{Computed tomography technique}

High-resolution computed tomography was performed with a Nanotom system manufactured by Phoenix X-ray. The system is equipped with a high-power nanofocused tube $(180 \mathrm{kV} / 15 \mathrm{~W})$ for a microstructural characterization of solar cells. The Nanotom of phoenix $X$-ray uses an X-ray cone beam creating twodimensional X-ray images while progressively rotating the sample step by step through a full $360^{\circ}$ rotation. These projections contain information on the position and density of absorbing object features within the sample. This accumulation of data is used for reconstruction of the volumetric data. The reconstructed 3D 
volume shows object features in gray values based on the differences in material density. It provides three-dimensional images at microscopic resolution [3]. In this work, computed tomography $3 \mathrm{D}$ images were generated by rotation of the sample over $360^{\circ}$ with a step size of $0.5^{\circ}$ around an axis, while taking a series of $2 \mathrm{D}$ pictures. Afterwards these images were combined to create a $3 \mathrm{D}$ volumetric representation of the structure using the reconstruction algorithm, which includes tools for geometry calibration, detector calibration, noise and beam hardening reduction. Voxel data segmentation and analysis was made by the "VG studio max" software package. Main parameters of the Nanotom system are shown in Table 1.

Table 1. Main parameters of Nanotom system.

\begin{tabular}{|c|c|}
\hline $\begin{array}{c}\text { Max. object size } \\
\text { (height/diameter) }\end{array}$ & $6 " \times 5 "$ \\
\hline Max. resolution & $<0.5 \mu \mathrm{m}(3 \mathrm{D})$ \\
\hline Nano focus tube & $180 \mathrm{kV} / 15 \mathrm{~W}$ \\
\hline Detector & $\begin{array}{c}12 \text { bits resolution } \\
2200 \times 2200 \text { pixels }\end{array}$ \\
\hline
\end{tabular}

The following parameters were used for solar cell scanning: Voltage $110 \mathrm{kV}$, Current 100 $\mu \mathrm{A}$. The position of the sample relative to the detector resulted in a voxel size (3D pixel) of $1.25 \mu \mathrm{m}$. The total scanning time for 1080 projections was 90 minutes.

\subsection{Materials}

Three different commercially available aluminum metal pastes (pastes designated $A$, $B$ and $C$ ) were investigated in order to find the differences in microstructure of the back contacts.
A standard industrial solar cell process was used, where screen printing of $\mathrm{Al}$ on the rear and $\mathrm{Ag}$ on the front was performed in the conventional manner with a 165 mesh screen and $250{ }^{\circ} \mathrm{C}$ drying temperature. In all cases neighboring multicrystalline wafers were used. Microstructural characterization of the $\mathrm{Al}$ and $\mathrm{Ag}$ bulk layers, concentration profile determination of glass phases in the as-fired $\mathrm{Al}$ and $\mathrm{Ag}$ paste, as well as a porosity investigation was performed.

Two drying temperatures $\left(250{ }^{\circ} \mathrm{C}\right.$ and 350 ${ }^{\circ} \mathrm{C}$ ) were chosen to examine its influence on the microstructure and composition of the aluminum pastes.

In order to obtain a higher resolution, all the samples were laser cut into small $10 \times 10 \mathrm{~mm}^{2}$ specimens and glued on small glass cylindrical bars.

\section{RESULTS AND DISCUSSION}

\section{1. Porosity and glass phase distribution in aluminum and silver metallic contacts}

X-ray CT was used for qualitative and quantitative analysis of internal features of screen printed metallic contacts. A strong contrast mainly exists between solid phases and the atmosphere where sufficiently great differences in atomic composition and/or density can be found.

Visualization of porosity and the distribution of different solid phases generally require pronounced variations in atomic composition. All elements presented in the Al and Ag layers have different atomic numbers: $\mathrm{Si}-14, \mathrm{Al}-$ $13, \mathrm{Bi}-83, \mathrm{Ag}-47, \mathrm{~Pb}-82$, thus it is possible to observe porosity and glass phase distributions.

Figure 1 shows 2D X-ray images of the three different Al pastes fired on top of the silicon wafer, showing the differences in
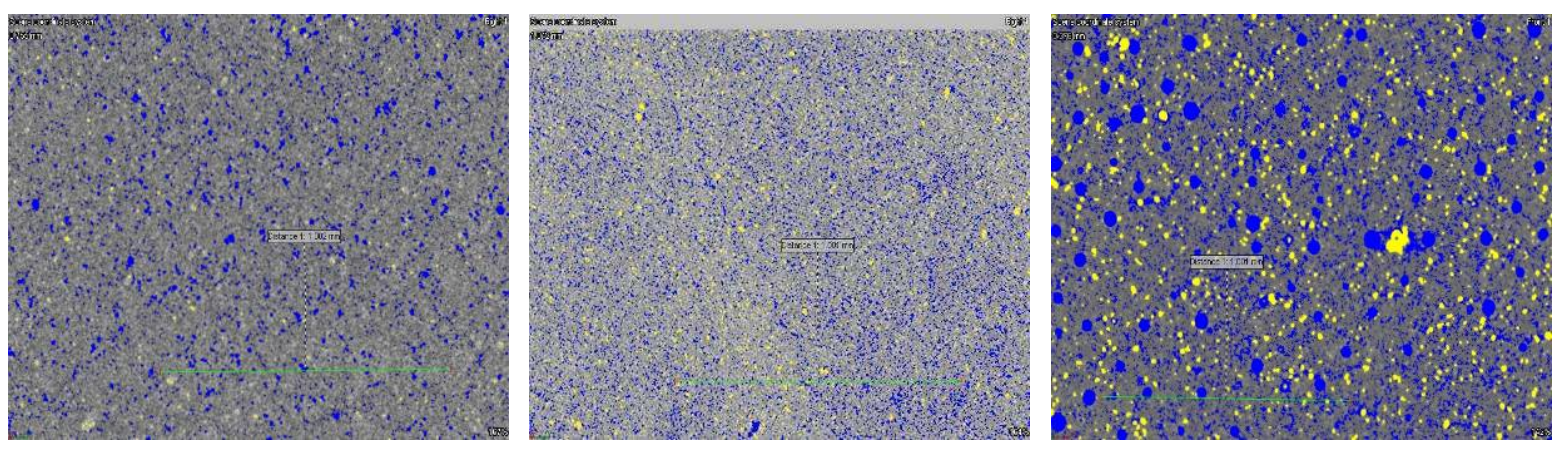

Figure 1. 2D X-ray image of the three Al pastes (A, B and C), screen printed and fired on top of the silicon wafer. Images show the differences in photographic density between different parts (yellow part: bismuth, blue: porosity, grey: $\mathrm{Al}$ and $\mathrm{Si}$ ). 
photographic density between different parts (yellow part: bismuth, blue: porosity, grey: Al and Si matrix). The samples were scanned perpendicular to the Al layer surface. Based on these images and a high contract difference between pores, bismuth glass and $\mathrm{Al} / \mathrm{Si}$ matrix it is possible to obtain quantitative information about the layers.

Table 2 represents a summary of the porosity and bismuth glass concentration for the aluminum layer. At least 5 different samples for each group of pastes were scanned, in order to obtain more reliable data. As can be seen from Table 2, all three Al pastes have different amounts of porosity and glass. Porosity results obtained by computed tomography show good agreement with the previous data, where the Al layer was investigated by Mercury intrusion porosimetry [4] .

Figure 2 shows the application of computed tomography for the silver front contact characterization.
A 2D X-ray image of the Ag layer (Fig. 2a), reveals the differences in photographic density between different parts (yellow part: porosity, red: lead glass, grey: silver). A cross section volumetric representation of the silver layer shows bismuth glass phase (red) and porosity (yellow) distributions (Fig. 2b).

A summary of the resulting data presented in table 2 was compared with the amount of bowing of the investigated samples, which was studied in the previous work [5]. It can be suggested that there is a relation between the porosity, glass phase concentration and the amount of bowing. For instance, in the case of aluminum pastes this relation can be formulated as follows: the higher the amount of porosity, the higher the bowing and the lower the concentration of glass, the lower the bowing is. However, this investigation is not complete, and some further tests will be performed. The effect of different Al paste type on the mechanical stresses in the layer is described in previous work [6].

Table 2. Summary of the porosity and bismuth glass fraction for aluminum and silver pastes, obtained by computed tomography

\begin{tabular}{|c|c|c|c|}
\hline Paste type & Porosity, $\%$ & Glass phase, $\%$ & Bowing, $\mathrm{mm}$ \\
\hline Aluminum paste A & 10 & 1.8 & 1.4 \\
\hline Aluminum paste C & 14 & 3.9 & 1.8 \\
\hline Aluminum paste B & 16 & 4.2 & 2.6 \\
\hline Silver paste & 7.5 & 6.5 & 4.7 \\
\hline
\end{tabular}

a)

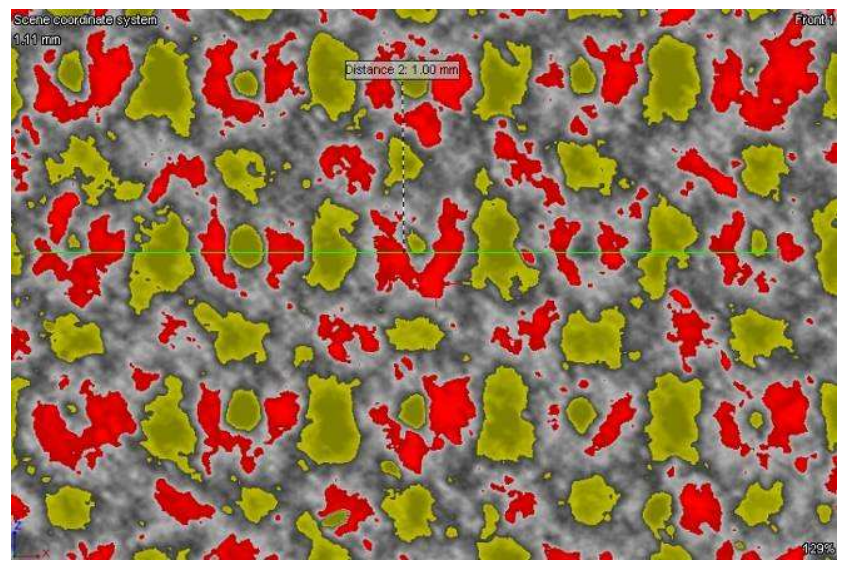

b)

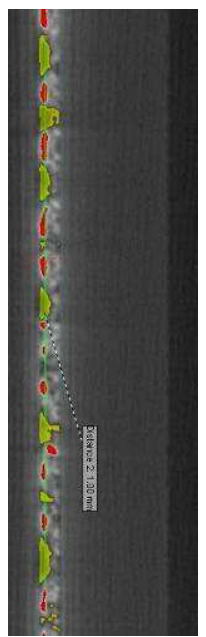

Figure 2. a) 2D X-ray image of the Ag layer, showing the differences in photographic density between different parts (yellow part: porosity, red: bismuth glass, grey: silver). b) Cross section volumetric representation of bismuth glass phase (red) and porosity (yellow) distributions 


\subsection{Defects imaging in Aluminum and Sil- ver layers.}

X-ray computed tomography scanning was performed on industrial multicrystalline solar cells with screen printed and fired aluminum paste $B$. As can be seen in Figure $3 a, C T$ scans revealed the presence of spherical voids inside the bulk Al layer. These voids have a homogenous and systematic distribution along the entire Al layer, indicating a processinduced nature of the defect. Figure $3 \mathrm{~b}$, represents an optical cross sectional image of the void, showing the absence of the eutectic and BSF layers underneath the void. Thus, it can be suggested that these defects affect electrical properties (as there is no BSF layer locally) and mechanical properties (resulting from the non-homogenous stress distribution) of the solar cell. Further investigations showed that the diameter of the voids and dimensions of the steel wires, used in screen printing process are identical.

The defects distribution pattern is also similar to the screen printing mesh, shown in Fig. 3c. Hence, it can be concluded that these voids

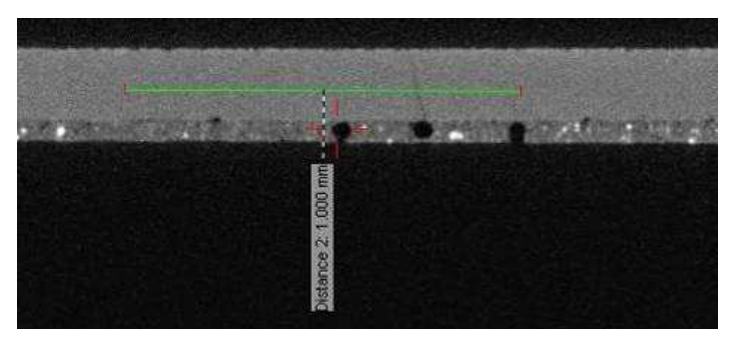

b)

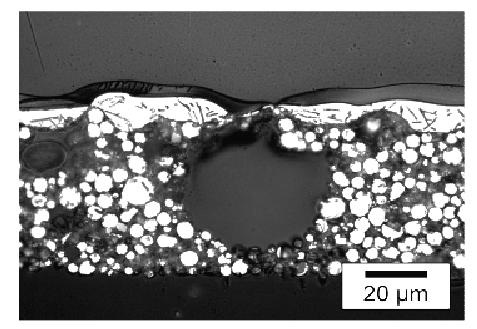

screen printing process-induced defects.

Two different drying temperatures $\left(250{ }^{\circ} \mathrm{C}\right.$ and $350^{\circ} \mathrm{C}$ ) and two screen printing mesh sizes (165 and 325) were used in order to investigate the nature and cause of the defects. CT scans of the samples with two screen mesh sizes did not show any differences. However, CT detected a significant change in the defects concentration between the samples with different drying temperatures, Fig.4. As can be seen, drying at $350{ }^{\circ} \mathrm{C}$ creates larger holes in a well defined pattern, thus producing a more porous layer. On the other hand, drying at $250{ }^{\circ} \mathrm{C}$ gives smaller holes and thus a denser Al layer structure.

The aluminum paste layer needs special care during the drying process (a slow heat-up ramp), otherwise volatilizing solvent can build cavities in the paste. These cavities produce unalloyed regions and porosity, which are correlated with the location of the screen mesh openings of the screen printing process.

Drying at $250{ }^{\circ} \mathrm{C}$ can be recommended as the most optimal temperature, creating fewer defects.

a)

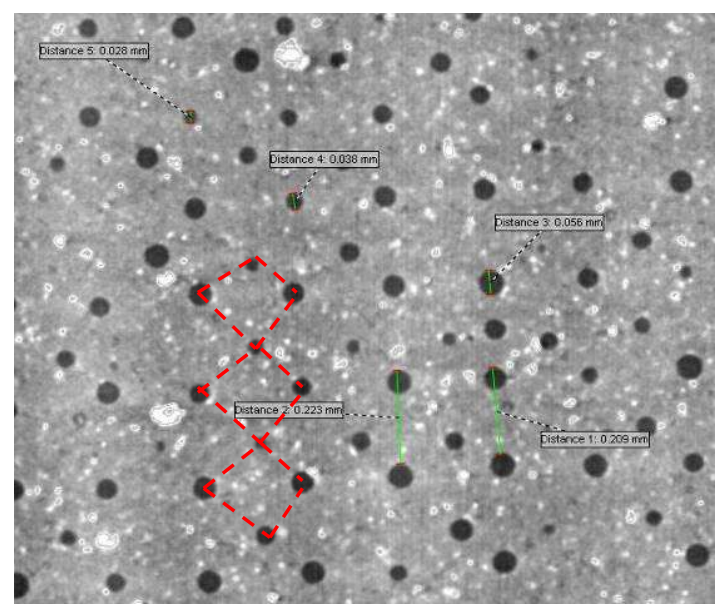

c)

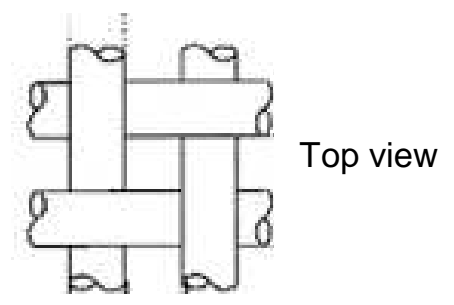

Cross section view

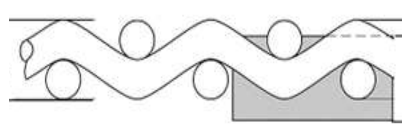

Figure 3 a) Cross section volumetric representation of the solar cell (upper image) and a 2D X-ray image of the Al layer (lower image), showing the screen printing process-induced defects (black part: voids, white: bismuth, grey: Al and Si). Dashed red line represents a reconstructed screen printing mesh. b) Optical image of the solar cell cross section showing the process-induced cavity. c) Industrial screen printing mesh, used for the application of metallic pastes [7]. 

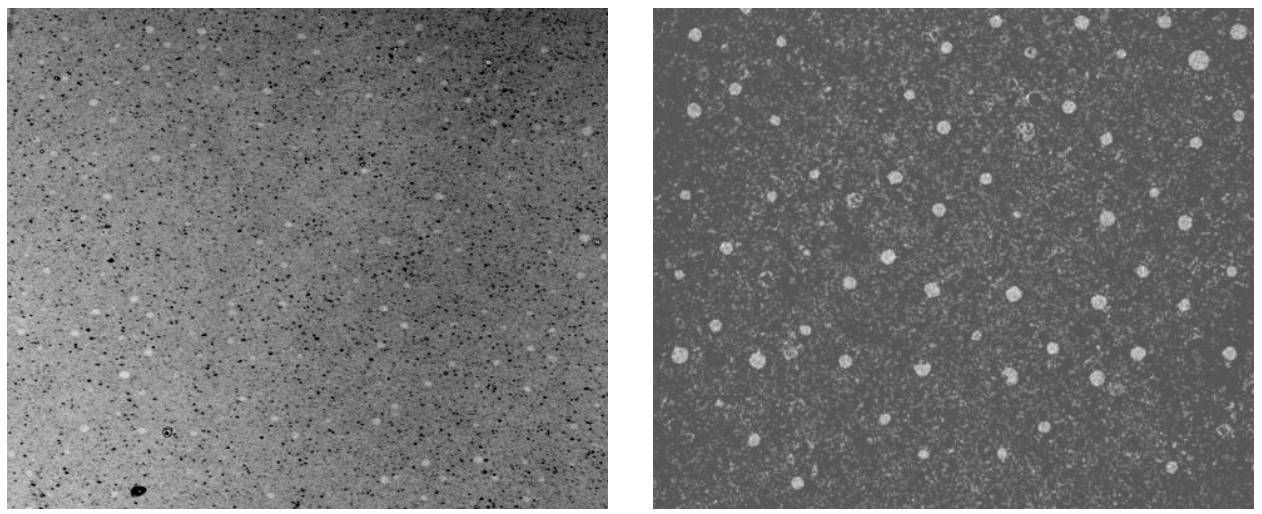

Figure 4. Effect of Al paste drying temperature on the formation of voids: left - drying at $250{ }^{\circ} \mathrm{C}$, right - drying at $350{ }^{\circ} \mathrm{C}$

Another example of a computed tomography investigation is shown in Figure 5, which shows the glass phase distribution and defects imaging in the silver front contact layer. As can be seen, the glass phase (corresponding to the red areas in Fig. 5a, b) concentrates at the $\mathrm{Si} / \mathrm{Ag}$ interface and at the outside of the $\mathrm{Ag}$ layer (Fig. 5a). A deep look inside the silver layer shows well defined parallel lines at the interface between $\mathrm{Ag}$ and $\mathrm{Si}$. It could be that these lines correspond to the local glass phase etching/penetration into the silicon wafer surface, thus creating a strong mechanical bonding. The nature of the parallel lines can be explained by the wire saw cutting process, which induces a damage layer at the silicon wafer surface.
It should be noted, that the distance between the red lines and the width of the lines show good agreement with wire saw cutting parameters (typical wire diameter is $150 \mu \mathrm{m}$ ) $[8,9]$. It is generally considered, that the damage layer removal process, by chemical etching, removes the cutting grooves, shown in Fig. 5c. However, CT images, obtained in this work, clearly demonstrate the presence of cutting grooves after firing of the silver contact. It can be suggested that residual microcracks serve as dominant areas for glass etching/penetration into the silicon wafer during the firing process. Thus, the etching process might open the residual microcracks and allow a preferential distribution of glass phases inside those cutting grooves. a)

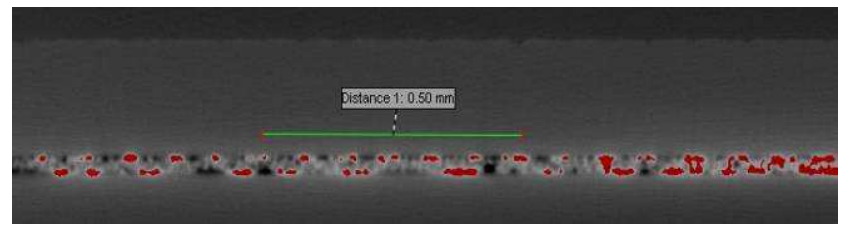

b)

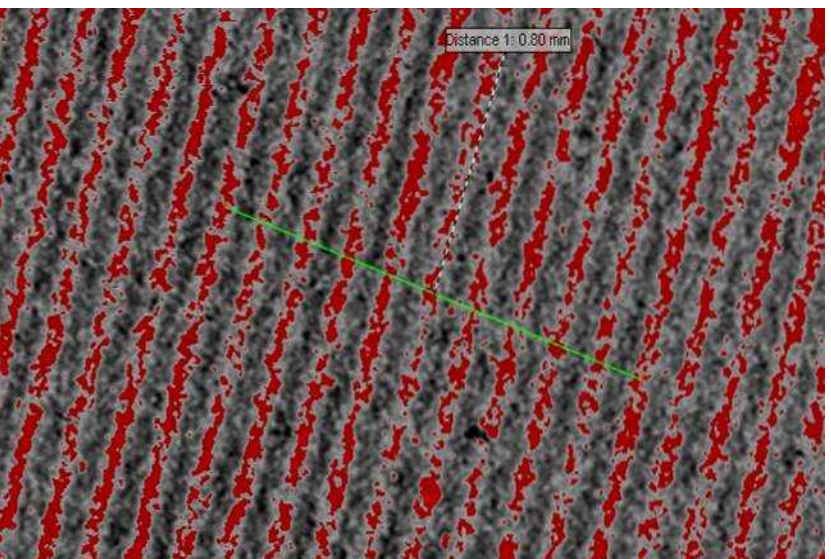

c)

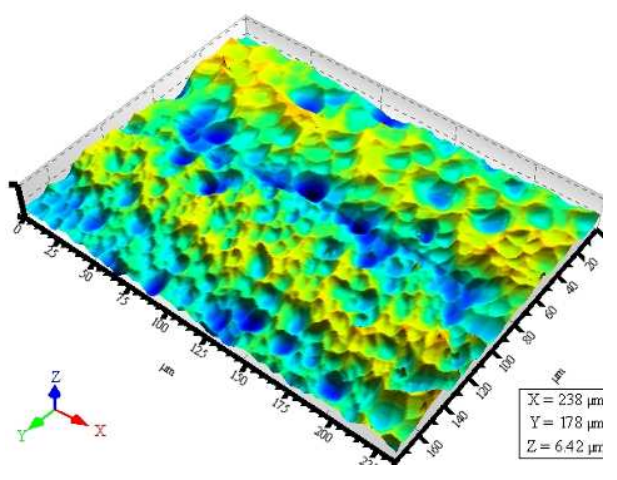

Figure 5. a) CT cross section image, showing glass distribution in silver layer b) CT image of the interface between silver layer and silicon wafer, showing a preferable distribution of bismuth glass (red part) in well defined parallel lines c) Confocal microscopy image of the silicon wafer, showing wire saw damage layer 
In order to prove this suggestion, some future experiments with mechanically polished wafers will be performed.

\section{CONCLUSIONS}

X-ray computed tomography is a powerful non-destructive observation technique that finds application whenever the evalutation of a material microstructure without causing damage is required. The use of this experimental technique in the field of solar cells, as presented here, is new and some preliminary results on the porosity and defects evolution were described.

X-ray 3D computer tomography was used in this work to investigate the internal architecture of the silicon solar cells. Resulting tomographic images clearly showed details of the distribution of bismuth glass and porosity in the $\mathrm{Al}$ and $\mathrm{Ag}$ contact layers. At the same time, 3D tomographic images revealed the presence of process-induced defects. Visualization of these defects is possible only by using computed tomography analysis. Hence, CT is an attractive non-destructive characterization tool with wide applications in solar cell industry. This work illustrates various examples of how CT can be used quantitatively to investigate the microstructure and the behavior of silicon solar cell metal contact layers.

Other studies based on tomographic analyses are planned as, for example, the effect of different processing parameters on microstructure and defects state of silicon solar cells.

\section{REFERENCES}

[1] General Electric Medical Systems CT HiLight Advantage Scanner System, Product specification B7980A, 1990.

[2] Standard Guide for Computed Tomography (CT) Imaging Designation: E 1441-91 American Society for Testing and Materials.

[3] xray.com/en/faq/index.html\#top

[4] V.A. Popovich, et al., Microstructure and mechanical properties of aluminium back contact layers Sol. Energy Mater. Sol. Cells 2010, in press.

[5] V.A. Popovich, T. van Amstel, I.J. Bennett, M. Janssen, I.M. Richardson; Microstructure and mechanical properties of aluminum back contact layers. In: Proceedings of $24^{\text {th }}$ (EPSEC) Hamburg, Germany, 2009.

[6] V.A. Popovich, A. Yunus, M. Janssen, I.M. Richardson, I.J. Bennett, Effect of microstructure and processing parameters on mechanical strength of multicrystalline silicon solar cells, IEEE, USA, 2010

[7] M.Hilali, Understanding and development of manufacturable screen printed contacts on high sheet-resistance emitters for low-cost silicon solar cells, 2005.

[8] H. J. Moller, Wafering of silicon crystals, phys. stat. sol. (a) 4659 , 2006.

[9] J. Li,, I. Kao,V. Prasad, Modeling Stresses of Contacts in Wire Saw Slicing of Polycrystalline and Crystalline Ingots: Application to Silicon Wafer Production, Transactions of the ASME 122 394, 2000. 